\title{
An IEEE 802.21-based Approach to Integrate Unidirectional Wireless Technologies into QoS-constrained Broadband Wireless Mesh Access Networks
}

\author{
Mathias Kretschmer \\ Fraunhofer FOKUS \\ Sankt Augustin, Germany
}

\author{
Gheorghita Ghinea \\ Brunel University \\ London, United Kingdom
}

\begin{abstract}
Wireless Mesh Networks (WMNs) research has matured in recent years and implementations are being tested around the world. In a WMN, participating nodes are automatically configured allowing them to communicate among their peers via multiple hops when no direct physical connectivity exists. This allows community networks such as Freifunk[5] to provide affordable Internet access to their communities by sharing a few gateway nodes. Increasing demand for multi-media content poses a challenge for the typical WMN routing protocols such as Optimised Link State Routing (OLSR) which lack Quality of Service (QoS) support. Although, numerous solutions have been proposed to introduce QoS awareness, those WMNs fail to meet commercial operator requirements in term of reliability and QoS guarantees. In this paper we propose a novel IEEE 802.21-based approach seamlessly integrating broadcast technologies such as Digital Video Broadcast (DVB) into a heterogeneous multi-radio WMN architecture in order to provide support for high-bandwidth multi-media services.
\end{abstract}

Keywords Heterogeneous technologies, QoS, IEEE 802.21, Wireless Mesh Network, MPLS, undirectional links

\section{Introduction}

The visibility of successful WMN deployments has raised interest regarding a commercial exploitation of this technology. Compared to traditional operator access networks, WMNs potentially offer easier deployment and flexible self-configuration at lower costs. For a WMN-type architecture to be considered as an alternative for an operator access network, it must meet similar requirements such as guaranteed QoS and high availability. QoS support for WMNs has received a lot of attention from the research community [10][1][19], but has yet to show its applicability in real world deployments.
Compared to the Freifunk-approach which relies on cheap off-the-shelf hardware, a Carrier-grade Wireless Mesh Access Network (CG-WMAN) may assume more reliable and higher performing multi-radio hardware yielding more stable and potentially orthogonal links. Thus, CGWMANs can be compared to a typical operator access network with two major limiting factors: the severe capacity constraints due to scarce wireless spectrum resources and temporarily varying link conditions. A modern operator access network needs to support triple-play services (web, video, voice) which emphases the need to support QoS guarantees and efficient resource utilization in a capacityconstrained network. Wireless cell resource utilization can be optimized by using the most suitable technology for a given payload. For example, the delivery of live multimedia content is facilitated by only one transmitter, hence the overhead of Media Access Control (MAC) protocols can be avoided. Unidirectional technologies such as Digital Video Broadcast - Terrestrial (DVB-T) offer a very efficient distribution medium for 1-to-N traffic and usually span across multiple mesh hops.

Typically, WMNs rely on the bi-directionality of links and can therefore not utilize Unidirectional Links (UDLs). For example, Ad hoc On-Demand Distance Vector Routing Protocol (AODV)[15] black-lists UDLs since they break the distance vector algorithm. What's more, most Mobile Adhoc Network (MANET) and WMN protocols can not differentiate between a faulty 802.11 link and an inherently unidirectional link provided by a unidirectional technology. In this paper we propose an Institute of Electrical and Electronics Engineers (IEEE) 802.21-based approach to seamlessly integrate UDLs into a heterogeneous CG-WMANs so that higher layer services can transparently utilize them when beneficial for a given payload or receiver distribution.

This paper is structured as follows. In the next section we discuss work related to our CG-WMAN architecture focusing on the support for UDLs. In the following section we compare two approaches to seamlessly integrate UDLs into the CG-WMAN and identify which protocols or com- 
ponents need to be adapted. In section four we present our approach to seamlessly integrate UDLs below the Network layer. The last section concludes this paper and gives and outlook on future work.

\section{Related Work}

The CG-WMAN architecture builds on proven technologies which have been extended to support heterogeneous wireless technologies. The core of the control plane builds on an extended version of IEEE 802.21, which allows for a hardware-independent and modular architecture design. The network management components such a topology discovery and path computation can be implemented as modules using the 802.21 messaging mechanism. This differentiates our approach from typical Network layer routing protocols, which integrate similar functionality in one protocol and are often agnostic to physical hardware capabilities.

\subsection{IEEE 802.21}

The main goal of IEEE 802.21 is to provide link layer intelligence for the upper layers. This should allow for a more intelligent decision making capability leading to more reliable and efficient handovers between heterogeneous networks. Besides mobile terminal hand-overs, functions of IEEE 802.21 can also be used for the management of heterogeneous networks such as security, emergency services and power management relying on the resilient messaging characteristics specified in the IEEE 802.21 standard. Each of those topics is studied within the sub-groups of the IEEE 802.21 working group with the target of preparing amendments to the standard.

IEEE 802.21 does not cover the actual mechanisms to perform a handover, but provides means to facilitate a handover. Hence, issues such as handover policies or security are omitted entirely. The considered access technologies comprise the IEEE 802 family, 3GPP and 3GPP2. The working group concentrates only on the realization of intertechnology hand-overs and does not cover intra-technology hand-overs, except for hand-overs between different extended service sets (ESS) of IEEE 802.11.

The scope of IEEE 802 focuses on link layer technologies and below. In order to support IEEE 802.21 in operator networks, work is being done in other standard bodies to support the deployment of 802.21. For example, the IETF MIPSHOP working group works on defining recommendations on how to discover an MIHF in Internet Protocol (IP)based networks[4]. Also, IP transport protocols such as Transmission Control Protocol (TCP) and User Datagram Protocol (UDP) areas being proposed as transports to exchange IEEE 802.21 messages.
The 2008, the IEEE 802.21 working group has initiated the $802.21 \mathrm{~b}$ study group to investigate issues concerning the use of IEEE 802.21 over unidirectional technologies. While the study group focuses on hand-overs, in this work, we focus on management of internal mesh interfaces, not access interfaces.

\subsection{21 CG-WMAN Amendments}

The CG-WMAN architecture design adopts the general IEEE 802.21 [6] architecture. The main difference between the IEEE 802.21 standard and the CG-WMAN architecture is the respective target application. IEEE 802.21 focuses on media independent hand-overs of mobile terminals, while the CG-WMAN provides solutions to manage heterogeneous wireless networks in a media independent fashion. The majority of the primitives defined by IEEE 802.21 can also be utilized for non-handover related purposes, such as managing local and remote radio technologies in a media independent manner. This characteristic is a main requirement for the $\mathrm{CG}-\mathrm{WMAN}$ architecture, which builds on IEEE 802.21 primitives where possible and extends those with mesh specific requirements.

As depicted in Figure 1, the Media Independent Handover Function++ (MIHF++) of the CG-WMAN extends the IEEE 802.21 Media Independent Handover Function (MIHF) with primitives specific to wireless network management, therefore the name Interface Management Function (IMF) has been chosen to reflect its responsibilities which go beyond Media Independent Handovers. This amendment to IEEE 802.21 provides a single interface for realizing Mobile Terminal (MT) hand-overs as well as building and managing a heterogeneous wireless networks.

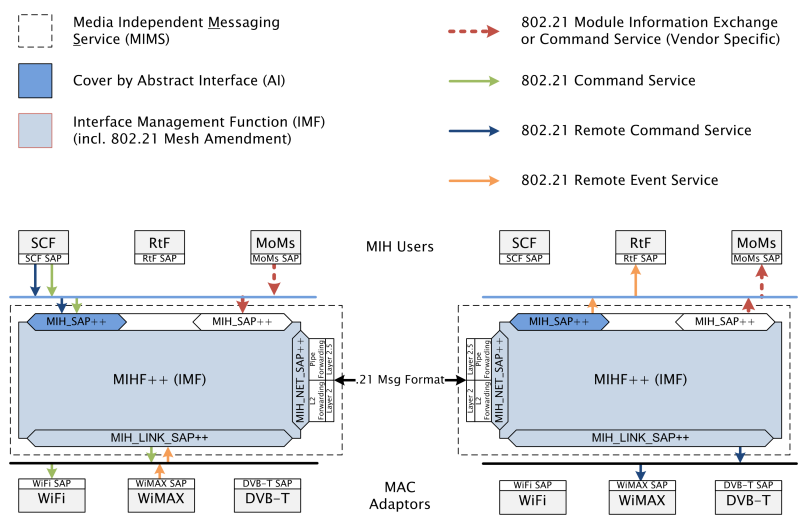

Figure 1. IMF extends the IEEE 802.21 MIHF by Module-to-Module Communication

In Figure 1, the dark colored MIH_SAP++ corresponds to abstract interface primitives of the CG-WMAN while 
white one corresponds to the module specific primitives. The primitives have been separated due to the fact that module specific primitives of the CG-WMAN are mainly related to wireless network management, while the $\mathrm{Ab}$ stract Interface (AI) provides a more generic interface for managing lower layers. In IEEE 802.21 the dark colored MIH_SAP++ would correspond to link events and commands and the white one would correspond to handoverrelated commands.

IEEE 802.21 does not foresee any direct communication between two remote higher layer entities via MIHF. It is assumed that higher layer entities are already aware of each other and rely on vendor specific means of communication, such as IP-based protocols. In the CG-WMAN architecture it is assumed that the IMF is aware of the higher layer entity responsible for a specific message coming from a remote IMF. This mapping of message identifiers to the responsible module is maintained via the following module registration procedure between a module and the IMF:

Each module provides a set of primitives. Combined, these primitives define the Service Access Point (SAP) of a module. Each primitive is identified by a unique message identifier (MID). During the registration process of a module to the IMF the module conveys the set of MIDs of its SAP. Thereby the IMF can forward an incoming message to the corresponding module by the MID. In this context, the MIDs can be seen as service identifiers where a module offers to provide the service identified by a specific MID.

Communication between two IMF instances is performed using the 802.21 message format. Message transport is realized via CG-WMAN management pipe whenever possible. If a pipe between the two IMF instances has not yet been established, a simple Data Link layer forwarding mechanism serves as a fall-back.

\subsection{Multi Protocol Label Switching}

Unlike hierarchical prefix routing which is typically associated with IP packet routing in the Internet, Multi Protocol Label Switching [18][17] tags packets with a 32 bit wide label based on which forwarding is then performed in intermediate routers, see Figure 2. Multi Protocol Label Switching (MPLS) operates at an Open Systems Interconnection (OSI) layer which is often referred to as Layer 2.5, between the data link and the network layer and MPLS labels are positioned between link layer headers and the headers of the encapsulated packet; usually a layer 3 protocol header, as shown in Figure 3. The name MPLS refers to its independence from both layer 2 and layer 3 protocols, which makes MPLS an interesting candidate for data forwarding in a WMN that integrates heterogeneous technologies such as IEEE-based ones and those developed by $3 \mathrm{GPP}$ or DVB, as well future emerging technologies.
MPLS paths are unidirectional, hence MPLS could be used on top of UDL without any need for modifications. Three bits in the MPLS header are designated for QoS prioritization and congestion notification which is especially important in a capacity-constrained network such as a CGWMAN.

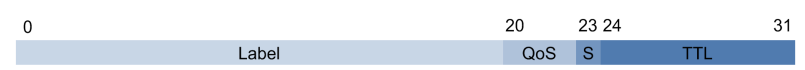

\section{Figure 2. MPLS Label Stack Entry (MPLS Shim Header)}

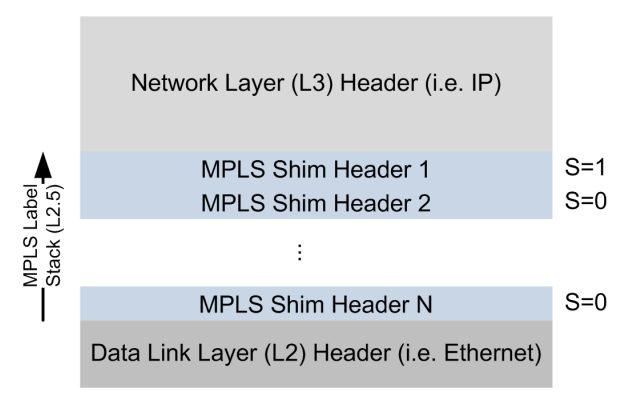

\section{Figure 3. MPLS Label Stack embedded into the protocol stack headers}

When a packet enters its ingress router, which is called Label Edge Router (LER) in MPLS terminology, the MPLS header is prepended to outgoing datagram. Or, in MPLS terminology, a label is pushed onto the label stack of the packet. Downstream routers simply look up the first label in the stack of the packet in a table that maps labels to outgoing interfaces and therefore next hop routers. Forwarding is then performed based solely on the table entry referenced by the label. In order to avoid scalability limitations of globally unique labels, intermediate routers generally rewrite the label of a packet to be forwarded with another label which has only local significance for the next hop. This exchange is referred to as label switching.

When a packet reaches its egress router in the MPLS network, the label is removed. Or, in MPLS terminology, the last label is popped from the label stack, and further packet handling must be performed based on the header information of the actual packet. MPLS can carry numerous traffic types, incl. IP, Asynchronous Transfer Mode (ATM), or IEEE 802.3(Ethernet)[13].

The paths which are given by the label based forwarding states in all routers between ingress and egress routers are called Label-Switched Paths (LSPs). LSPs are conceptually similar to virtual connections in ATM, although unlike ATM, MPLS supports variable packet lengths. To set up LSPs in IP networks, Constraint-based Routing Label 
Distribution Protocol (CR-LDP) or Resource ReSerVation Protocol - Traffic Engineering (RSVP-TE)[3] have been defined by the Internet Engineering Task Force (IETF). In 2003 a decision[2] was taken by the IETF to obsolete CRLDP in favor of RSVP-TE. MPLS can be used to support and enforce Traffic Engineering decisions when forwarding data along Path Computation Element (PCE) computed paths.

\subsection{MPLS Fast Reroute (FRR)}

MPLS Fast Reroute (FRR) [14] can provide protection against link breakage in a LSP by a backup path that circumvents the broken links. Each LSP can have a dedicated backup path, as depicted in Figure 4, or all LSPs sharing network segments can also share the same backup path, see Figure 5. Maintaining backup paths introduces higher protocol overhead, but provides faster recovery in case of node or link failures when compared to higher layer protocol recovery, which is particularly useful for real-time traffic. In a capacity constrained WMN backup paths might block precious capacity needed for regular payload traffic and the trade-offs need to be considered carefully.

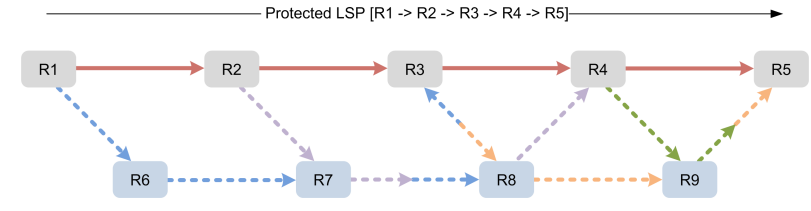

Figure 4. MPLS One-to-One Backup

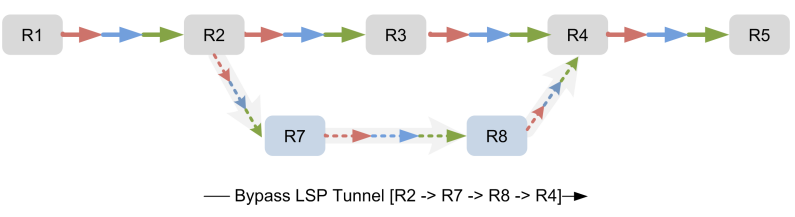

Figure 5. MPLS Facility Backup

\subsection{MPLS Signalling and Unidirectional Links}

MPLS path setup as well as triggering FRR needs to be addressed when using MPLS over UDLs, since RSVP-TElike approaches, for example, rely on bidirectional physical connectivity between the path starting node, the intermediate nodes and the endpoint node. Triggering FRR is typically - in wired networks - implemented via a layer two event indicating a link failure. The transmitter on a wireless UDL has no means to detect a reception problem on the receiver side. Hence, to support FRR over UDL, a monitoring system capable of handling UDLs is required which could then trigger FRR to forward traffic around a broken link [11].

\subsection{Unidirectional link routing}

Unidirectional Link Routing (UDLR) [7] provides a mechanism to emulate full bidirectional connectivity between all nodes that are directly connected by a unidirectional link. The receive-only node uses a tunneling mechanism to forward link layer datagrams back to send-only nodes via a separate bidirectional IP connectivity. A typical tunneling protocol used in combination with UDLR is Generic Routing Encapsulation (GRE)[8], which is an IP protocol. Since the tunnel encapsulates the data link layer frames, UDLR is considered transparent to higher layer protocols.

UDLR may cause problems with mechanisms such as Internet Protocol, Version 6 (IPv6) Duplicate Address Detection (DAD) which sends out a Neighbor Solicitation packets to detect if other nodes already use the probed address. The standard DAD mechanism has been designed for regular link-local broadcast domains, such as an Ethernet segment. It assumes that only other nodes will respond to this solicitation packet, while in the UDLR case, it will receive its own solicitation packet and may therefore assume the address to be already allocated.

UDLR uses Dynamic Tunnel Configuration Protocol (DTCP) embedded in its downstream UDL to announce to receive-only nodes at which IP address the tunnel endpoints for the return channel can be reached. Each node with a send-only interface can signal where the tunnel endpoints for a specific unidirectional link can be found. Since UDLR transparently provides bidirectional link layer connectivity between the nodes, normal routing protocols could be used without a need for modifications incorporating unidirectional link support.

However, the transparent return link provided by UDLR poses a major problem especially for carrier-grade networks since the higher layer protocols are not aware of the special nature of such link which often crosses numerous heterogeneous networks and wireless cells until reaching the tunnel endpoint. It is therefore not possible to perform per-hop resource reservations or link monitoring. Hence including UDLR links in a QoS-constrained path computation algorithm would requires major adaptations. DTCP allows for multiple tunnel endpoint addresses to be announced, but can not control, which is eventually used by a receive-only node. Return link failures can not be detected by UDLR, since return channels are implemented via state-less GRE encapsulation. 


\subsection{Bidirectional Routing Abstraction}

Bidrectional Routing Abstraction[16] uses a reversed distance vector algorithm to detect UDLs in MANETs. Bidrectional Routing Abstraction (BRA) provides an abstraction layer so that higher layer protocols only see bidirectional links. Support for QoS-constraint path computation suffers from similar implications due the hidden heterogeneity of the return link as described for UDLR.

\section{Approach}

In this chapter we first discuss possible approaches to seamlessly integrate Unidirectional Links into a CGWMAN as internal mesh interfaces, taking the architecture defined by the CARrier grade wireless MEsh Network (CARMEN) project[9] as a basis. The minimum requirement on a participating node is to provide one receivecapable and one transmit-capable interface. In most cases, a node will be equipped with bidirectional and unidirectional interfaces. Our proposed solution will be optimized for both cases. We will highlight advantages and discuss the consequences of the approaches. The chosen approach will then be described in detail.

\subsection{Possible Approaches}

The goal of seamlessly integrating UDLs in a CGWMAN is to allow higher layer services to transparently utilize UDLs when they are beneficial to address physical radio or deployment conditions, given payload characteristics or receiver distributions. Two approaches come into close consideration, namely the below the network layer or the at the network layer approach. Both approaches have advantages but also introduce implications. The CARMEN project has made the following architecture design decisions which need to be considered when integrating UDLs:

- Control plane and hardware abstraction are based on IEEE 802.21

- Unidirectional interfaces can be identified and their capabilities described

- Network management and routing are based on a centralized approach

- Individual traffic flows are aggregated into QoS-aware pipes

- Pipes are unidirectional resources established between any pair of nodes

- In the multicast case, a pipe is extended into a 1-to-N tree

\subsection{Below the Network Layer Approach}

This approach considers an integration at and above the Data Link layer and addresses UDLs where they occur as a result of MAC protocol design decisions or physical characteristics of the radio technologies. In order to support the QoS-aware pipe concept, MPLS may be used on the data plane to provide QoS-aware forwarding of user data along centrally computed path. MPLS is usually considered a layer 2.5 technology, hence the approach proposed here would not be a pure Data Link layer approach, but rather a Below the Network Layer approach. It would be transparent to higher layers, and through the use of MPLSbased forwarding allow for QoS-constrained routing, unlike approaches such as BRA[16] or UDLR[7].

The use of MPLS on the data plane complements the support for heterogeneous technologies, with an IEEE 802.21-based control plane providing support for local as well as remote management of heterogeneous nodes and their radio interfaces.

Routing is traditionally a Network layer task. Although, simple routing algorithms such as Spanning Tree can be found in lower layers, as well. The IEEE 802.11s working group has taken a similar approach by integrating a variant of AODV into the 802.11s MAC layer and thus can provide IEEE 802.11 mesh clouds transparently to the network layer. In the centralized CG-WMAN approach, QoSconstraint routing state is maintained at the centralized Path Computation Element, which is independent from any routing protocol that might be operational among the network nodes. Using the routing state available at the PCE, management traffic can be sent via source routing or explicitly configured MPLS LSPs. Hence, no additional routing protocol is required.

MPLS path setup is typically implemented via higher layer protocols such as RSVP-TE. In the case of the centralized approach, no distributed routing state exists in the network. Hence, an explicit source routing mechanism would be required for the path setup procedure. A concept similar to RSVP-TE using its explicate source routing object could be implemented via explicit source routing among the IMFs along the path to be configured using special tunneling Media Independent Handover (MIH) messages. Such messages could therefore be processed at each hop's Routing Function which in turn would configure the MPLS forwarding state.

\subsection{At the Network Layer Approach}

An integration at the Network layer would mean to address Data Link layer related requirements at a layer that naturally integrates heterogeneous Data Link layer capabilities. The support for UDLs could be seen as an exten- 
sion of that concept. In the case of IP-based protocols the data link technologies are often assumed to provide bidirectional means of communication, hence an integration of UDLs would require modification to proven IP-related protocols, such as interface auto-configuration, neighbor discovery, routing or flow signaling.

Alternatively, UDLR could be deployed to provide tunneled return channels for UDLs to provide virtually bidirectional interfaces. Since UDLR assumes a rather static network configuration regarding, for example, the return channel tunnel endpoints, an additional mechanism would be required to allow for UDLR self-configuration.

Following the UDLR concept, an IP-tunneled return channel provided by UDLR would be detected as one hop by the routing protocol even though it may span across a large set of heterogeneous links and wireless cells. Hence proper QoS allocation can not be performed for the return channel, without taking the links forming the tunnel into account. This would require the path and wireless cell resource computation module to be aware of sub links within a logical link.

An alternative could be to only use UDLR return links for management traffic to avoid using them for regular allocated data plane pipes. Modifications to the routing protocols would be required to detect and black-list UDLR return links when computing data plane pipes.

As a mesh network has no default routing hierarchy, a routing protocol is required in order for non-neighboring nodes to communicate with each other even for management purposes. This is required independently from the centralized QoS-constraint Path Computation function. Under the assumption that a CG-WMAN node always has at least one bidirectional link, UDLs could be ignored at the cost of potentially lower mesh connectivity, as shown for BRA. This is the default behavior of common WMN protocols such as AODV or link state protocols such as Open Shortest Path First (OSPF). In this case, nodes without a bidirectional interface could not be supported.

To support RSVP-TE over UDLs the Explicit Route Object could be used. This concept may need to be extended so that this object could hold the downstream and upstream path. The upstream path might take a different route but would need to traverse the same nodes in order for the RESP message to be processed properly by all nodes along the path.

\subsection{Integration Below the Network Layer}

Conceptually, an integration either at or below the Network layer is possible. An integration below the Network layer seems to be the more natural fit, since the unidirectional links can be seen a Data Link layer or Physical layer phenomenon. In addition, Data Link layer protocols are usually less complex compared to higher layer protocols. Hence the required modifications are less intrusive.

Path computation in the below-the-network-layer approach is straight forward, since UDLs are fully integrated and represented as regular logical links, while in the network layer approach UDLs would require special handling with a varying complexity depending on the level of integration.

The below-the-network-layer approach is a solution for a CG-WMAN that seamlessly integrates UDLs so that higher layer protocols and services can run without modifications.

The CARMEN project has evaluated a fully centralized vs. a centralized but distributed (i.e. OSPF-like) approach to perform QoS-constrained routing. As a first approach CARMEN has decided to deploy a fully centralized routing approach where QoS-accounting and routing resp. MPLS path computation is performed in the gateway nodes and path state is then pushed into the intermediate mesh nodes along the path from the source towards the destination and vice versa.

The below-the-network-layer layer approach will be designed to fit into the CARMEN CG-WMAN architecture, which means that the following components need to be evaluated for compliance with UDLs:

- Hardware Abstraction \& Heterogeneous Technologies

- Control Plane Messaging

- Link \& Flow Monitoring

- Topology Discovery and Maintenance

- Path Computation

- Path Signaling

- Data Plane Forwarding

Figure 6 depicts a high-level architecture view of a CGWMAN node. The control plane is managed by the Interface Management Function (IMF) which builds on and extends the MIHF defined in the IEEE 802.21 standard. The Message Transport component provides various IEEE 802.21 compatible transport mechanism required by the CG-WMAN management components. The Forwarding Fucntion implements data plane forwarding following the concepts of MPLS.

\subsection{Heterogeneous Technologies}

The CG-WMAN architecture integrates heterogeneous technologies via MPLS on the data plane while the control plane relies on an extended set of IEEE 802.21 primitives and remote messaging. Additional primitives to extend 


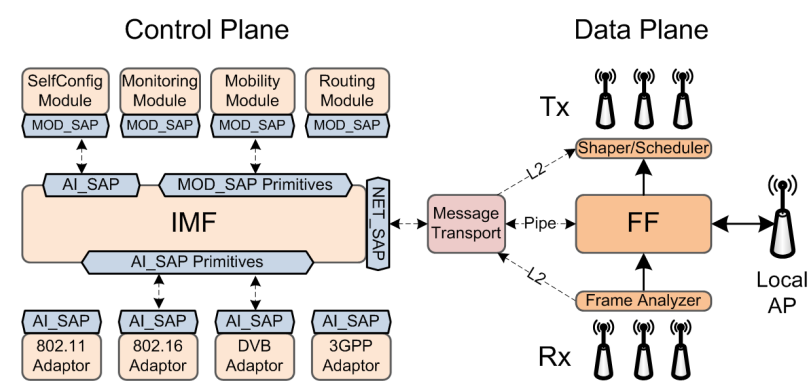

\section{Figure 6. The IEEE 802.21-based architecture of the CG-WMAN}

IEEE 802.21 beyond support for Media Independent Handover have been defined by the CARMEN project forming the so-called Abstract Interface. Some of those additions have been proposed for standardization which has led to the forming of the $802.21 \mathrm{~b}$ study group to address broadcast technology support. A recently started study group investigates the configuration of wireless network devices. Additional primitives defined within this work extend the CARMEN-proposed primitives where needed to add native UDL support.

While the majority of technologies provides bidirectional communication, the minimum requirement for a technology to be integrated is the ability to address and transmit a frame from node $\mathrm{A}$ to node $\mathrm{B}$ or to receive a frame on a specific interface while being able to identify the sender address. Also, each node must be equipped with, at least, one transmit-capable and one receive-capable interface. This could be one bidirectional interface, two unidirectional interfaces or a combination thereof.

The AI abstracts the capabilities of common radio technologies in order to provide a common interface to the upper layers. The parameters covered include:

- Channel frequency

- Channel bandwidth

- Transmit power

- Modulation and Coding Scheme properties

- MAC scheduler properties

- Directionality

- Antenna properties

An important parameter within the Scheduler Properties is the operational mode, which describes the different modes that common wireless technologies may implement:

- Infrastructure mode, where a Base Station controls a set of Subscriber Stations
- Ad-Hoc mode, where all stations operate in the same mode without a coordinator

- Broadcast mode, where only one sender is active on a channel and an arbitrary number of receivers may be present

Together with the directionality information, the mode parameter helps to determine if a technology implements bidirectional, or unidirectional communication. This can also be used to detect anomalies such as, for example, IEEE 802.11 links that seem to only communicate unidirectionally due to unstable radio conditions.

\subsection{Control Plane Messaging}

The CG-WMAN control plane builds on and extends the IEEE 802.21 messaging service which has been extended by a module-to-module communication component, thus implementing an Remote Procedure Call (RPC) framework based on IEEE 802.21 primitives. IEEE 802.21 does not specify a specific message transport for inter-node communication, but suggests that data link communication as well network layer communication may be used. The ID of the messaging function, the so-called MIHF ID, of a node may be determined via various algorithms, but must be ensured to be unique. Within this work, it is computed as a hash of a node's interface addresses. Possible collisions will be detected and resolved by the Carrier-Grade Gateway (CGW), which will ensure that associated Carrier-Grade Mesh Points (CMPs) use unique MIHF IDs. In order to address the various communication needs of the CG-WMAN management components, four complementary transports have been defined:

- Link Local - Link-local communication via Link Layer Multicast

- Management Pipes - Dedicated MPLS LSPs between communicating nodes

- Flooding - Flooding based message forwarding with smart pruning

- Link Vector - Explicit source routing

\subsection{Link Local Multicast Messaging}

Link local messaging can be used to reach all nodes which are direct neighbors on any of the sending node's interfaces. According to the 802.21 standard, the destination MIHF ID is set to Multicast. This mechanism is typically used by the 802.21 Information Service. 


\subsection{Management Pipe}

A pair of CG-WMAN pipes with traffic class MANAGE$M E N T$ can be established between any two nodes. Due to the centralized design, this means that usually a pair of Management Pipes will exist between the CGW and any CMP managed by it. Disjoint MPLS backup paths could be configured to increase the connectivity in the case of intermediate pipe segment failures. This is the most efficient and therefore also the default communication mechanism in the CG-WMAN

\subsection{Flooding}

This transport implements a flooding mechanism where each node forwards an incoming message if it is not the destination as specified in the MIH header destination TLV. The flooding scope is limited by a Time to live (TTL) and a 'message already seen' list maintained by each node. A message is identified by it's source and destination MIHF ID as well as the transaction ID. If a forwarding node knows a more efficient transport to the destination, it will stop flooding and use that transport.

This mechanism should only be used if no other transports are available. In the centralized approach, this should only be the case in the event of a management pipe failure, during initial boot strapping or network partitioning.

\subsection{Link Vector}

This transport provides an explicitly source routed mechanism to forward a message to a destination. Such a mechanism is crucial to implement an RSVP-TE-style path setup in a centralized CG-WMAN where routing state is only available at CGW node. It can also be utilized as a lightweight solution to send MPLS FRR triggers to Point of Local Repairs (PLRs), potentially via multiple disjoint routes in parallel. A vector element is of the type LinkID which consists of the source as well as the destination link layer addresses of the described link. Hence, messages can be send from any node to a remote node without the need for a neighbor discovery mechanism such as Address Resolution Protocol (ARP). Therefore, this mechanism also supports UDLs.

If the vector only contains one element, this mechanism provides an explicit link local delivery, where the local sending as well as the remote receiving interface are specified via the LinkID. In this case, the remote address my be a Multicast address to reach all nodes associated with the source interface. This mechanism can, for example, be used to organize local broadcast domain tasks.

\subsection{Compatability with IEEE 802.21 and UDL support}

Link Local Multicast Messaging has been defined in the IEEE 802.21 standards document for use by the Information service which provides broadcast-style link-local delivery. Hence, it can used on bidirectional and transmit-capable unidirectional interfaces.

The Management Pipe and Flooding mechanisms provide an equivalent to a Network Layer transport allowing for network-wide communication. If present, the Management Pipe transport will be preferred. Both mechanisms support UDLs.

The Link Vector mechanism is related to source routing in the IP protocol family. While it could be used as the general transport mechanism, it introduces a higher overhead and is therefore only beneficial for special communication needs. In such a case the Link Vector is specified by the source node. Since the IEEE 802.21 header can not be extended with transport specific TLVs, an encapsulation primitive is defined, which consists of a regular MIH header, an encapsulation specific TLV holding the link vector and a TLV holding the original MIH primitive's packet, see Figure 7.

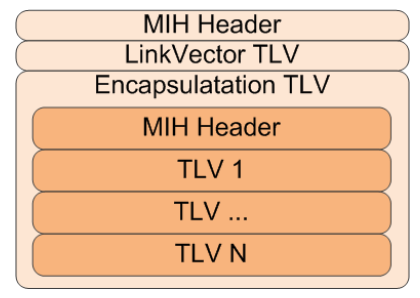

Figure 7. The MIHEncapsulation Primitive consists of an MIH header, a LinkVector TLV and a TLV holding an encapsulated 802.21 primitive

The transport-specific TLV can now be interpreted by all nodes along the specified path. At the ultimate hop, the encapsulation is removed and the original primitive's MIH packet is delivered to the IMF which can dispatch it to the registered MIH User or MAC Adaptor. This mechanism is similar to other encapsulation or tunneling mechanisms, such as GRE. It does not require any modifications to the existing IEEE 802.21 protocol headers, while the encapsulated messages while just be ignored by a regular 802.21 MIHF. This mechanism supports UDLs.

\subsection{MPLS Fast Reroute}

MPLS FRR provides a backup facility for complete LSPs or segments thereof. Backup path computation and 
signaling are similar to the procedures defined for regular LSPs and therefore no additional considerations are required to support UDLs.

Multiple strategies to trade-off fail-over reliability against the capacity blocked for fail-over paths have been discussed within the CARMEN project and in the literature. Those consideration can be applied to UDLs, as well. This aspect is out of the scope of this work.

The detection and signaling of the occurrence of failover events is covered by the Monitoring component which also addresses measurement and evaluation over UDL. The delivery of fail-over events, however, needs to be investigated for UDL support, since the a fail-over event would need to be signaled to a PLR, which in many cases might not be identical with the CGW and therefore no default management pipe exists. To support a quick signaling in a network without routing state, the signaling path should be pre-computed. This could be done by installing management pipes between the PLR all nodes along the protected paths that might detect and trigger the fail-over event.

However, if the link failure is caused by external interference, it might impact the communication in the radio vicinity of the detecting node. Hence the installed management pipe might be impacted, as well. To increase the chance of a quick and successful delivery of the event at the PLR, multiple disjoint path may be used in parallel. This mechanism relies on Link Vector messaging to deliver the message along a precomputed path. When configuring the LSP backup state, the CGW may also compute disjoint path from each protected node to the PLR. Those path will be stored as link vectors and can be used to send the event to the PLR. Those precomputed paths can also easily be adapted by the CGW if required due to topology changes.

\subsection{Link \& Flow Monitoring}

Monitoring support for UDLs may require modifications to existing concepts such as the use of passive receiver side monitoring where possible, see [11]. To configure, operate and maintain a CG-WMAN, the monitoring component is expected to provide the following set of events to the centralized network controller:

- Transmitting interface detected

- Transmitting interface disappeared

- Physical Link Associated

- Physical Link Lost

- Pipe QoS Violation detected

- Pipe QoS Violation predicted

- Pipe Inactivity detected

\subsection{Topology Discovery \& Maintenance}

Topology Discovery and Maintenance in a CGW-centric CG-WMAN is initiated and controlled by gateway nodes (CGWs) which aim at discovering the mesh nodes in their vicinity and also form routing areas or clusters to establish a two-layer hierarchy in order to keep topology changes local. The proposed topology discovery and maintenance mechanism is a continuous process that supports bidirectional as well as unidirectional technologies. The optimization goal for the Topology Maintenance function is to maintain a well balanced mesh, with redundant connections for each CMP towards the $\mathrm{CGW}$ which should be of similar distance.

\subsection{Path Computation}

Path computation in the CG-WMAN is performed by a centralized entity, similar to the Path Computation Element concept. The path computation component might be located at each CGW or at a remote location. In the latter case, each CGW will act as a proxy for the remote entity. The path computation algorithm is based on the Dijkstra algorithm used by most link-state routing protocols. In order to support capacity-constrained routing, the path computation module will also maintain a data base of already allocated resource per link and consider wireless cell resource allocations. Since logical links always describe unidirectional resources, UDLs will appear in the link state table as regular links and considered by the routing algorithm. This table of logical links is generated and maintained by the Topology Maintenance module, and consists of the optimized subset of all possible physical links within a routing area.

\subsection{Multicast Tree Computation}

In order to more efficiently utilize longer range broadcast cells, the CG-WMAN routing algorithm is extended to address the trade-off between in-mesh forwarding of traffic versus the use of longer range broadcast cells, see [12]. This becomes especially important for the distribution of multicast traffic with a larger number of receivers. Single source 1-to-N multicast routing within a CG-WMAN with overlay cells can be configured in different ways depending on operator policies, receiver distribution and QoS requirements.

\subsection{Path Signaling}

The objective of the Path Management Protocol (PMP) is to signal paths between two arbitrary CMPs within a CGWMAN which may span across UDLs. This includes the negotiation of local scope MPLS labels to avoid label collisions at downstream nodes. 
To implement an RSVP-TE-like path signaling protocol in a CG-WMAN without routing state available at each hop, the use of explicit source routing is seen as the most viable solution. In order to support UDLs, the source routing vector elements need to specify both ends of a link, the source address and the destination address. This is because with unidirectional technologies, local address resolution or neighbor discovery protocols can not be relied on. The CGWMAN describes its logical links via LinkIDs which consist of the source and the destination link layer address. Using the Link Vector forwarding mechanism and the LinkIDs as a link description, any path in the CG-WMAN can be explicitly specified and therefore LSP state can be configured.

\subsection{Data Plane Forwarding}

The CG-WMAN relies on MPLS for forwarding within the mesh network. This mechanism is inherently unidirectional. To support UDL it is required that for each link or LSP segment, the source and destination link layer address are known at the upstream node, since neighbor discovery or address resolution protocols can not be relied on in the case of a UDL. This requirement is addressed by PMP which signals the path using a vector of LinkIDs, which describe a link using its source and destination link layer address.

\subsection{Multicast Support}

MPLS supports 1-to-N multicast fan-out at each LabelSwitched Router (LSR) effectively turning a point-to-point LSP into a tree. To support UDL the same considerations as for unicast LSP forwarding apply and no extra adaptations are required.

\section{Conclusion and Future Work}

An integration of UDLs below the Network layer seems most suitable for the our heterogeneous CG-WMAN and its centralized architecture. The control plane tasks can be mapped onto an extended set of IEEE 802.21 primitives while QoS-constrained data plane forwarding can rely on MPLS. The advantage of is approach is that rather more complicated Network layer protocols such as Mobile IP do not need to be modified.

Future work will focus on the efficient utilization of DVB overlay cells in a CG-WMAN. Instead of relying on the same rather cell-local metric used for mesh links, we will investigate modern decision making algorithms to balance between the requirements of the payload, the topological distribution of the receivers and the costs of overlay cell resources compared to mesh links.

\section{Acknowledgment}

The research leading to these results has received funding from the European Community's Seventh Framework Programme (FP7/2007-2013) under grant agreement no 214994. The views and conclusions contained here are those of the authors and should not be interpreted as necessarily representing the official policies or endorsements, either expressed or implied, of the CARMEN project or the European Commission.

\section{References}

[1] N. Abu-Ali and H. Hassanein. Wlc30-5: Statistical delay budget partitioning in wireless mesh networks. pages 1-5, 27 2006-dec. 12006.

[2] L. Andersson and G. Swallow. The Multiprotocol Label Switching (MPLS) Working Group decision on MPLS signaling protocols. RFC 3468 (Informational), Feb. 2003.

[3] D. Awduche, L. Berger, D. Gan, T. Li, V. Srinivasan, and G. Swallow. RSVP-TE: Extensions to RSVP for LSP Tunnels. RFC 3209 (Proposed Standard), Dec. 2001. Updated by RFCs 3936, 4420, 4874, 5151, 5420.

[4] G. Bajko. Locating IEEE 802.21 Mobility Services Using DNS. RFC 5679 (Proposed Standard), Dec. 2009.

[5] F. Community. http://www.freifunk.net. Accessed 22May-2009.

[6] A. De La Oliva, A. Banchs, I. Soto, T. Melia, and A. Vidal. An overview of ieee 802.21: mediaindependent handover services. Wireless Communications, IEEE, 15(4):96 -103, aug. 2008.

[7] E. Duros, W. Dabbous, H. Izumiyama, N. Fujii, and Y. Zhang. A Link-Layer Tunneling Mechanism for Unidirectional Links. RFC 3077 (Proposed Standard), Mar. 2001.

[8] D. Farinacci, T. Li, S. Hanks, D. Meyer, and P. Traina. Generic Routing Encapsulation (GRE). RFC 2784 (Proposed Standard), Mar. 2000. Updated by RFC 2890.

[9] http://www.ict.carmen.eu. Accessed 22-apr-2009.

[10] V. Kone, S. Das, B. Y. Zhao, and H. Zheng. Quorum - quality of service routing in wireless mesh networks. In ICST Conference on Heterogeneous Networking for Quality, Reliability, Security and Robustness (QShine), 2007. 
[11] M. Kretschmer, C. Niephaus, and G. Ghinea. QoSaware flow monitoring and event creation in heterogeneous MPLS-based wireless mesh networks supporting unidirectional links. In 9th IEEE Malaysia International Conference on Communications 2009, Kuala Lumpur, Malaysia, 2009.

[12] M. Kretschmer, C. Niephaus, and G. Ghinea. Towards QoS Provisioning in a Heterogeneous CarrierGrade Wireless Mesh Access Networks Using Unidirectional Overlay Cells, volume 22 of Lecture Notes of the Institute for Computer Sciences, Social Informatics and Telecommunications Engineering. Springer Berlin Heidelberg, 2009.

[13] L. Martini, E. Rosen, N. El-Aawar, and G. Heron. Encapsulation Methods for Transport of Ethernet over MPLS Networks. RFC 4448 (Proposed Standard), Apr. 2006. Updated by RFC 5462.

[14] P. Pan, G. Swallow, and A. Atlas. Fast Reroute Extensions to RSVP-TE for LSP Tunnels. RFC 4090 (Proposed Standard), May 2005.

[15] C. Perkins, E. Belding-Royer, and S. Das. Ad hoc OnDemand Distance Vector (AODV) Routing. RFC 3561 (Experimental), July 2003.

[16] V. Ramasubramanian and D. Mosse. Bra: A bidirectional routing abstraction for asymmetric mobile ad hoc networks. IEEE/ACM TON 16(1):116-129, 2008.

[17] E. Rosen, D. Tappan, G. Fedorkow, Y. Rekhter, D. Farinacci, T. Li, and A. Conta. MPLS Label Stack Encoding. RFC 3032 (Proposed Standard), Jan. 2001. Updated by RFCs 3443, 4182, 5332, 3270, 5129, 5462, 5586.

[18] E. Rosen, A. Viswanathan, and R. Callon. Multiprotocol Label Switching Architecture. RFC 3031 (Proposed Standard), Jan. 2001.

[19] H. Zhou, C. Huang, Y. Cheng, and G. Wang. A new multi-metric qos routing protocol in wireless mesh network. volume 1, pages 459-467, april 2009. 